\title{
GÉNERO Y EDUCACIÓN
}

\section{Experiencias e iniciativas para prevenir la Violencia de Género entre adolescentes}

"Educando en Igualdad". Plan de Igualdad del IES Castillo de Fatetar, Espera (Cádiz)

\section{Por: Zoraida Pérez Sabio}

Coordinadora de Igualdad del IES

Castillo de Fatetar de Espera (Cádiz)

e-mail: zperez9@gmail.com

\section{Resumen}

El objeto del presente artículo es dar a conocer las experiencias e iniciativas en materia de prevención de violencia de género llevadas a cabo con el alumnado del IES Castillo de Fatetar a través de los objetivos marcados en el Plan de Igualdad del Centro. El proyecto en el que se enmarcan estas iniciativas, "Educando en Igualdad", se ha diseñado para conseguir cambios en las actitudes, conocimientos y habilidades del alumnado adolescente; con el fin de eliminar los mitos e ideas erróneas subyacentes al fenómeno de la violencia de género; y de capacitar al alumnado para detectar y reconocer el maltrato físico, psicológico y sexual.

\section{Palabras clave}

Plan de Igualdad, Adolescencia, Experiencias, Talleres, Prevención, Violencia de género.

\section{Abstract}

The purpose of this article is to present the experiences and initiatives carried out with students from IES Castle Fatetar in the prevention of domestic violence through the targets set in the Plan for Equality Center. The project is called "Educating for Equality". It has been designed to bring about changes in attitudes, knowledge and skills of teenage students; The aim of the project is to dispel myths and misconceptions underlying the phenomenon of gender violence; and train students to detect and recognize the physical, psychological and sexual abuse. 


\section{Keywords}

Equality Plan, Adolescence, Experiences, Workshops, Prevention, Gender Violence.

\section{Introducción}

"Incorporar la perspectiva de género a la educación supone cimentar las bases para la igualdad real y efectiva, y se muestra como el mejor medio para impulsar el proceso de transformación social y modificación de las estructuras sexistas y discriminatorias" (I Plan Estratégico para la igualdad de mujeres y hombres en Andalucía 2010/2013, 29)

El reconocimiento del papel crucial que la educación puede y debe desempeñar para fomentar la auténtica igualdad y para prevenir la violencia de género, es hoy generalizado. En los últimos años se han puesto en marcha algunas medidas educativas dirigidas a la consecución de la igualdad y a la prevención y protección integral contra la violencia de género desde el ámbito educativo. Una de las medidas adoptadas para conseguir hacer realidad el principio de igualdad entre sexos es el desarrollo de Planes de Igualdad en los Centros educativos, en los que la formación e intervención del profesorado de forma activa en los procesos de cambio hacia la igualdad, se muestra como un factor clave para poder operar transformaciones educativas y sociales deseables, ya que tanto a través de sus enseñanzas y estrategias como de los valores que transmitan van a influir en el alumnado respecto a la formación de su identidad de género y por consiguiente en las relaciones afectivas que se establezcan entre el alumnado.

Es cierto, desde las aulas se debe hacer algo, pero también es cierto que la prevención de la violencia de género es una labor de toda la sociedad, en especial del entorno familiar y de los medios de comunicación. En la medida en la que se realice la coordinación, sensibilización e implicación de todos los agentes que conforman la comunidad educativa, los resultados serán más favorables y permitirán, además de hacer reflexionar al alumnado y a sus familias sobre los aspectos básicos de la igualdad de género, crear un clima de colaboración 
que genere "la necesidad de construir modelos coherentes con los valores de respeto y de igualdad entre las personas que conviven en el contexto familiar" (Tomé et al. 2006:46).

En este sentido, diseñar y aplicar el Plan de Igualdad en un centro educativo es una tarea compleja, que abarca muchos ámbitos y aspectos distintos (Moreno, 2013). Pero el objetivo fundamental que se debe establecer es el de movilizar al alumnado para reflexionar sobre los contenidos culturales y los valores en los que han sido educados y que siguen teniendo un marcado carácter androcéntrico, sexista y estereotipado (Rodríguez, 2004); y sobre todo, diseñar nuevas vías de intervención educativa que permitan "descubrir ante la juventud dichas situaciones discriminatorias y trabajar las erróneas atribuciones sexistas que utilizan para justificarlas" (De la Peña et al. 2011:7, 2으 parte).

\section{2. "Educando en Igualdad": Plan de Igualdad del IES Castillo de Fatetar}

El proyecto "Educando en Igualdad" surge en el curso 2011/2012 con el objetivo de fomentar un cambio en las actitudes individuales, conocimientos y habilidades sociales de un alumnado con edades comprendidas entre 12 y 18 años, procedente de un entorno rural, en el que siguen vigentes estructuras sociales desiguales, jerarquizadas y estereotipadas.

Tras la realización de un primer diagnóstico mediante encuestas ${ }^{1}$ en relación a la socialización del alumnado y sus familias sobre el uso de lenguaje sexista, estereotipos de género y responsabilidades y tareas familiares, nos encontramos un escenario bastante incierto, ya que sus respuestas reflejaron una gran aceptación de prejuicios y estereotipos sexistas, factores que alimentan la lacra social que supone la violencia de género y la desigualdad.

Teniendo en cuenta esta situación, se decidió hacer con el Plan de Igualdad una de las líneas prioritarias y visibles en el Proyecto de Centro del IES Castillo de Fatetar, centrando una parte muy significativa de su acción educadora en la igualdad entre hombres y mujeres, poniendo en marcha distintas propuestas de trabajo para dar un carácter coeducativo a las prácticas educativas cotidianas con iniciativas y estrategias tales como:

1 Las encuestas para el diagnóstico del Plan de Igualdad pueden consultarse en: http://www.juntadeandalucia.es/educacion/webportal/abaco-portlet/content/66e34810-6dd5-41e8-88d2$\underline{\text { b1fdd15ac551 }}$ 
- Incorporación de actividades de sensibilización en materia de igualdad en el Plan de Acción Tutorial.

- Creación de un espacio en el centro dedicado a la coeducación: tablón de Igualdad.

- Integración de la coeducación como eje transversal en todas las materias, así como la contribución de las mujeres a los diferentes campos del conocimiento, del arte y de la cultura a lo largo de la Historia.

- Realización de campañas de sensibilización a través de la convocatoria de concursos y ginkanas en fechas concretas.

- Coordinación y colaboración con el AMPA "IES Castillo de Fatetar" para la realización de proyectos de coeducación y la solicitud de talleres relacionados con esta temática subvencionados por la Junta de Andalucía, y de esta manera potenciar la incorporación de las familias a este proyecto.

- Realización de talleres sobre educación afectivo-social y prevención de la violencia de género en fechas determinadas, como el 8 de marzo (Día Internacional de la Mujer) o el 25 de Noviembre (Día Internacional Contra la Violencia hacia las Mujeres).

- Creación de un blog de Igualdad ${ }^{2}$ que permitiese visibilizar a toda la comunidad educativa cada una de las actividades en materia de igualdad realizadas en el Centro y de esta manera contribuir a la igualdad real de mujeres y hombres.

En la elaboración del Plan de Igualdad se plantearon los siguientes objetivos:

- Sensibilizar a la comunidad educativa de la necesidad de la coeducación en el ámbito educativo para conseguir una igualdad real en la formación entre hombres y mujeres.

- Conocer los factores sociales y las conductas que justifican la discriminación sexual.

- Impregnar toda la práctica educativa de los valores de coeducación.

\footnotetext{
2 "Educando en Igualdad". Blog coeducativo del IES Castillo de Fatetar, Disponible en : http://igualdadiescastillodefatetar.wordpress.com/
} 
- Posibilitar la creación de un ambiente que propicie en la comunidad educativa la materialización de los valores de tolerancia y la igualdad en la diversidad.

- Hacer reflexionar al alumnado sobre las limitaciones que los estereotipos sexistas representan para la realización de su vida personal, profesional y laboral, mediante la construcción de un nuevo modelo de identidad libre de las limitaciones del género.

- Usar el lenguaje de forma no sexista o coeducativa por parte de toda la comunidad educativa.

- Establecer colaboraciones con instituciones, organismos y asociaciones del entorno, a fin de conseguir líneas comunes de actuación.

- Promover todo tipo de actividades que lleven a una mayor sensibilización sobre la Violencia de Género.

\section{Talleres para prevenir la violencia de género entre adolescentes}

En los talleres de prevención diseñados, además de trasladar al alumnado la necesidad de establecer relaciones afectivas construidas desde el respeto y la igualdad entre hombres y mujeres, también se ha querido aportar al resto de la comunidad educativa (profesorado, madres y padres/familia), la información y estrategias necesarias, relacionadas con las actitudes y creencias que están presentes entre el alumnado.

En cada una de las fases de las que constan los talleres se les solicita ayuda y colaboración a las familias del alumnado, implicándolas en su desarrollo de forma activa, aportando y compartiendo experiencias y sugerencias ante el necesario cambio social que supone la erradicación de la violencia de género.

Los talleres están dirigidos a todo el alumnado del centro, de 1ㅇ a 4o de ESO y constan de las siguientes fases:

\subsection{Sensibilización y prevención de la violencia de género}


Fase previa a la realización de los talleres que permite sensibilizar al alumnado en esta problemática y que tomen conciencia y se posicionen activamente contra la violencia de género.

Hace dos años, en el curso 2011/2012, se realizaron las siguientes actividades de sensibilización durante las sesiones de tutoría:

- Realización y pegada de carteles con mensajes alusivos a la prevención de la violencia de género.

- “Árbol de las víctimas de la violencia de género". El alumnado investigó casos reales de violencia de género que se habían producido durante ese año y, de esta manera, se pudo comprobar que la violencia de género se puede producir en cualquier edad o situación socioeconómica. Con esta información se realizó un homenaje simbólico, escribiendo el nombre de las víctimas en palomas de papel que posteriormente se colocaron en un árbol del mismo material.

Durante el curso 2012/2013 se quiso dar un paso más a la mera sensibilización sobre el tema a nivel de tutoría y se convocó el "I Concurso de Carteles Contra la Violencia de Género IES Castillo de Fatetar" ${ }^{3}$. La participación del alumnado en este concurso fue elevada y los trabajos aportados fueron muy variados, en originalidad, calidad o creatividad artística; pero a fin de cuentas, todos transmitieron valores de igualdad y de repulsa hacia la violencia de género. En el pasado curso escolar se convocó la segunda edición del concurso ${ }^{4}$ y la respuesta del alumnado superó de nuevo todas las expectativas.

\subsection{Realización de los talleres}

La impartición de los talleres tiene lugar en torno al 25 de Noviembre: Día Internacional Contra la Violencia de Género. La temporalización estimada de los mismos es de una hora por curso y nivel, por lo que durante esa jornada se altera el horario habitual del Centro.

\footnotetext{
${ }^{3}$ Los carteles pueden consultarse en la siguiente dirección: http://igualdadiescastillodefatetar.wordpress.com/2012/11/23/carteles-ganadores-del-i-concurso-escolar-decarteles-contra-la-violencia-de-genero-ies-castillo-de-fatetar/

4 Los carteles pueden consultarse en la siguiente dirección:

http://igualdadiescastillodefatetar.wordpress.com/2013/11/24/carteles-ganadores-del-ii-concurso-escolar-decarteles-contra-la-violencia-de-genero-ies-castillo-de-fatetar/
} 
Los talleres consisten en el desarrollo de diferentes dinámicas para trabajar distintos aspectos relacionados con la violencia de género. Evidentemente, es una propuesta adaptada al contexto y a la realidad de mi Centro, por lo que es probable que en otro Centro en el que se quisiera intervenir tuviera que adaptarse, dependiendo del tiempo del que se disponga y del nivel de conocimiento del alumnado sobre el tema.

\subsubsection{Objetivos}

El objetivo general y prioritario de estos talleres es reflexionar y tomar conciencia sobre las causas y procesos que llevan a situaciones de desigualdad entre hombres y mujeres y a la prevención de la violencia de género.

Este objetivo general se complementa con otros más específicos, que son los que siguen:

- Vivenciar el camino de la igualdad entre géneros.

- Fomentar la construcción de una identidad individual no sexista y no violenta.

- Favorecer la adquisición de habilidades para enfrentar las situaciones de maltrato.

- Potenciar relaciones igualitarias y la solidaridad con las víctimas de la violencia de género.

\subsubsection{Metodología}

En los talleres se hace uso de técnicas metodológicas en las que intervenga activamente todo el alumnado asistente, con actividades tanto individuales como cooperativas, que permitan generar la participación y reflexión del alumnado y que sea capaz de promover el cambio en sus ideas previas. Se utilizan diferentes dinámicas para animar el debate adaptadas a los diferentes cursos en los que se interviene, como la lluvia de ideas, el role playing, el debate grupal o la reflexión individual.

En el desarrollo de los talleres es importante tener en cuenta el adecuado uso del lenguaje por parte del alumnado, de manera no sexista y discriminatoria, como objetivo a 
trabajar de manera transversal y que permitan crear actitudes y aptitudes para hacer un uso no sexista del lenguaje. En este sentido, se aportan soluciones lingüísticas que traten de evitar el contenido sexista que pudiera surgir, para que los mensajes y reflexiones que se emitan se realicen desde una perspectiva de igualdad. Además, se pueden hacer propuestas de cambio con las alternativas que se consideren más adecuadas al contexto en el que se inscriben, esperando contribuir a la disminución del sexismo lingüístico y por ende a la erradicación de la discriminación de género en la sociedad.

\subsubsection{Estructura}

En líneas generales, los talleres se han desarrollado sobre el mismo eje estructural, variando actividades según el nivel al que van dirigidas. El esquema de los talleres es el siguiente:

a. Visionado de un corto sobre la violencia de género. Son cortos adaptados a los cursos a los que se dirige el taller y les permite reflexionar y hacer una primera toma de contacto con la problemática planteada. Los cortos utilizados en nuestros talleres han sido los siguientes: El maltrato 'sutil', ${ }^{5}$, No te confundas ${ }^{6}$ y Piel'.

b. Dinámicas de parejas o grupales. Durante el desarrollo de esta actividad se anima al alumnado participante a colaborar en el desarrollo de las diferentes dinámicas, que se detallan a continuación:

- Dinámica de presentación por parejas (preferiblemente mixtas), en la que deben reflexionar sobre el maltrato y el buen trato. Una vez formadas las parejas, se les da un tiempo aproximado de 5 minutos para intercambiar opiniones o pareceres sobre lo que

\footnotetext{
${ }^{5}$ Corto escrito y dirigido por Diego Jiménez, con ilustraciones de Sonia Sanz Escudero. Se puede consultar en la siguiente dirección: https://www.youtube.com/watch?v=0y9zJ5J2bWA

${ }^{6}$ Forma parte de los 11 capítulos de la teleserie Apuntes, en los que se abordan diferentes temas que preocupan a los adolescentes. Está rodada por el grupo de teatro Embeleco del IES Sierra de las Villas de Villacarrillo (Jaén), bajo la dirección de Vicente Nieto. Se puede consultar en la siguiente dirección:

http://www.youtube.com/watch?v=WIXiCIZ1zmQ\&feature=share\&list=PL2OayO879B5I5YyndwILvCV1WwZJ5yPIG\&i $\underline{\text { ndex }=4}$

${ }^{7}$ Spot que forma parte de una campaña contra la violencia de género realizada por el ministerio de Sanidad, Política Social e Igualdad en el año 2012. Se puede consultar en la siguiente dirección http://www.youtube.com/watch?v=FSmkwm8L9bA\&list=PLrHu4SOEsuan8apU7e2wjTJ6GPaeYBNye\&feature=share
} 
- $\quad$ piensan o sienten cuando se enteran de que una persona ha sido tratada sin respeto, propiciándole un daño físico o psicológico. Pasados los 5 minutos, cada participante es presentado/a por su pareja comentando también su opinión al respecto. Durante el turno de presentaciones se anotan todas las opiniones en la pizarra, que permite crear una definición de "maltrato" por parte de todo el alumnado. Una vez que está definido el maltrato, se les propone el reto de encontrar ideas de buen trato colocándolas al lado de las anteriores, de esta manera el grupo al completo crea una definición de "buen trato".

- Juego de roles e identidades. Esta dinámica grupal consiste en la asignación de valores, positivos o negativos, a una serie de adjetivos estereotipados ${ }^{8}$ asociados tradicionalmente al género masculino y femenino. Dependiendo del nivel al que se dirija la dinámica, los agrupamientos podrán variar desde el pequeño grupo hasta el gran grupo. También se deberá adaptar la forma de realizar el listado de estereotipos, en el caso de 1으 y 응 de ESO, se les facilita el listado de estereotipos, sin embargo, el alumnado de 3ㅇ y 4으 de ESO lo realizarán en forma de "lluvia de ideas". Cuando la lista está elaborada se les pide que asignen un valor ${ }^{9}$ a cada uno de los estereotipos.

Para terminar esta dinámica, se hace una puesta en común con el planteamiento de preguntas como:

- ¿ ¿Os sentís identificados con el estereotipo que se os asigna?

- ¿Por qué se ha dado un valor u otro?

- ¿De qué manera influye, en vuestra vida cotidiana, un valor negativo o positivo?

- ¿ ¿A través de qué o de donde creéis que se transmiten estas ideas estereotipadas?

- ¿Qué pasa cuando un chico o una chica expresa un estereotipo que no está asociado a su género? ¿Se le trata bien/mal?

O

8 Dulces, sentimentales, débiles, fuertes, racionales, delicados/as, responsables, impulsivos/as, valientes, ordenados/as, emocionales, independientes, irresponsables, derrochones/as, indefensos/as, competitivos/as, deportistas o agresivos/as.

9 Los valores a asignar a cada estereotipo deben decidirlos con antelación. Pueden utilizar símbolos o cualquier tipo de lenguaje gráfico que les sea cercano, propios de redes sociales o de mensajería móvil (emoticonos, + o -, flechas ascendentes o descendentes, etc.) 
- ¿ ¿Hay algún estereotipo que se utilice como insulto hacia alguno de los géneros?

c. Definición de la violencia de género y los tipos de violencia hacia la mujer. Esta parte del taller es más expositiva, es decir, en este caso la responsable del proyecto les transmite esta información, con ayuda de una presentación. En próximos talleres será el propio alumnado el que indague y busque información al respecto, plasmándolo posteriormente en cartulinas o en formato digital, ya que así la asimilación de conceptos y el aprendizaje es significativo.

d. A continuación se realiza un visualizado del corto Sin pensarlo dos veces ${ }^{10}$, con el fin de analizar las fases del maltrato, así como los mitos y falsas creencias sobre el tema. Tras el visionado del corto se realiza un debate grupal planteando preguntas del tipo:

- ¿ ¿Os parece que controlar y dominar a una chica es una forma de ejercer la violencia?

- ¿Creéis que a un chico que es maltratador se le ve venir desde el principio de la relación?

- ¿Qué os parece el comportamiento del chico?

- ¿ ¿Por qué la golpea?

- ¿Es la forma más adecuada de solucionar los conflictos?

- ¿ ¿Creéis que la violencia es un asunto privado donde no hay que entrometerse?

e. Audición, análisis y reflexión de una canción ${ }^{11}$ relacionada con la temática tratada en el taller. Consiste en la audición activa de la canción seleccionada y el análisis del mensaje que pretende transmitir su letra. Para ello se realiza una puesta en común grupal a través de una serie de preguntas del tipo:

- ¿De qué trata la canción?

\footnotetext{
${ }^{10}$ Corto de la directora Chus Gutiérrez. Se puede consultar en la siguiente dirección: http://www.chusgutierrez.es/directora/detalle/obra/cortos-es-segmento-sin-pensarlo-dos-veces/

${ }_{11}$ Las canciones seleccionadas para los talleres fueron: Salir corriendo de Amaral, En tu ventana de Andy y Lucas, Que nadie de Manuel Carrasco y Malú.
} 
- ¿Creéis que existe algún tipo de violencia? ¿Cuál?

- ¿ ¿Hay expresiones discriminatorias o que indiquen estereotipos o prejuicios?

- ¿ ¿Habíais pensado antes en las canciones desde este punto de vista?

\section{f. Lectura colectiva y posterior reflexión de un cuento ${ }^{12} 0$ cómics ${ }^{13}$ sobre la violencia} de género. En el desarrollo de esta dinámica se hace uso de la técnica de Role Playing. Para la reflexión posterior a la lectura y dramatización se les puede guiar con preguntas del tipo:

- ¿ ¿Qué os parece esta situación? ¿Puede ser real?

- ¿Cómo actuaríamos en caso de ser las personas protagonistas?

- ¿ ¿Qué haríamos si esta situación de maltrato le ocurriera a un amigo o amiga?

Por último, todos los talleres terminan con el visualizado de la presentación realizada con todos los carteles participantes en los_Concursos de Carteles Contra la Violencia de Género del Centro, de los que se ha hecho mención en el punto 3.1 de este artículo.

\subsection{Fase de consolidación}

Con esta fase se pretende concebir la realización de estos talleres de una manera integral. Aunque inicialmente se diseñaron como una intervención puntual, la experiencia nos ha demostrado que las reflexiones que se inician en los talleres no han de quedarse ahí, sino que han de tener continuidad para poder consolidarse en cambios reales. En este sentido el Blog de lgualdad del centro cumple un papel esencial, junto con la realización de otros talleres coeducativos $^{14}$, a lo largo del curso y su inclusión como contenido específico en determinadas materias, como Educación para la Ciudadanía de 3o de ESO o Proyecto Integrado de 4을 de ESO. Por último, se destacan las iniciativas llevadas a cabo desde el Plan de Acción Tutorial o el programa Escuela Espacio de Paz, en las que se trabaja fundamentalmente la resolución pacífica de conflictos, valores importantísimos en la prevención de la violencia de género.

\footnotetext{
12 Puede servirnos de ejemplo el cuento Arturo y Clementina de Adela Turín.

13 Puede servirnos de ejemplo el cómic Pillada por ti editado por la Secretaría de Estado de Igualdad. Puede consultarse en esta dirección:

http://www.msssi.gob.es/ssi/violenciaGenero/publicaciones/comic/docs/castellano.pdf
}

14 Talleres organizados desde el Plan de Igualdad del centro (8 de Marzo), por otras instituciones públicas o asociaciones sin ánimo de lucro. 


\section{Conclusión}

La valoración del desarrollo de estos talleres por parte de toda la Comunidad Educativa ha sido muy positiva, ya que se ha apreciado un alto grado de implicación, receptividad y de preocupación por el tema. El alumnado ha mostrado mucho interés y ha participado activamente en todas las actividades y talleres planteados en el Centro a lo largo de todos estos años, demostrando que la educación es el mejor camino de cara a la prevención, y que ellos y ellas son elementos clave para erradicar la violencia en el futuro.

\section{Referencias bibliográficas}

I Plan Estratégico para la igualdad de mujeres y hombres en Andalucía: 2010/2013. Instituto Andaluz de la Mujer.

DE LA PEÑA, M., LUZÓN, J.M., RAMOS, E. y RECIO, P. (2011): Sexismo y Violencia de Género en la Juventud Andaluza. Instituto Andaluz de la Mujer

MORENO LLANEZA, Ma Antonia (2013). Queremos coeducar. [on line] Avilés: Centro de Profesorado y Recursos de Avilés-Occidente. Recuperado de: http://blog.educastur.es/marianmoreno/files/2013/06/queremoscoeducar-2.pdf

RODRÍGUEZ MARTÍNEZ, Carmen (Comp.) (2004): La ausencia de las mujeres en los contenidos escolares. Buenos Aires: Miño y Dávila ed.

TOMÉ, A., LOMAS, C., SIMÓN, E., SOLSONA, N., BALLARÍN, P. y BLANCO, N. (2006). Guía de Buenas Prácticas para Favorecer la Igualdad entre Hombres y Mujeres en Educación. Sevilla: Consejería de Educación, Junta de Andalucía. 